\title{
Finite-element analysis of the nonlinear propagation of high-intensity acoustic waves
}

\author{
C. Campos-Pozuelo \\ Instituto de Acústica, CSIC, Serrano, 144, 28006 Madrid, Spain
}

B. Dubus

Institut d'Electronique et de Microélectronique du Nord, département ISEN, UMR CNRS 9929, 41 boulevard Vauban, 59046 Lille Cedex, France

\author{
J. A. Gallego-Juárez \\ Instituto de Acústica, CSIC, Serrano, 144, 28006 Madrid, Spain
}

(Received 23 October 1997; accepted for publication 7 March 1999)

\begin{abstract}
This paper deals with a three-dimensional numerical procedure based on the finite-element method for the modeling of finite-amplitude progressive acoustic waves. The method can predict the nonlinear propagation of acoustic fields produced by sources of arbitrary geometry. Based upon a perturbation method, a second-order analytical study is developed and the numerical procedure is formulated. Basic equations are derived and their ranges of validity established. The analytical validation of the numerical development is presented. An experimental validation is also carried out for the nonlinear acoustic field radiated by a stepped-plate transducer. (C) 1999 Acoustical Society of America. [S0001-4966(99)00407-5]
\end{abstract}

PACS numbers: 43.25.Cb [MAB]

\section{INTRODUCTION}

High-intensity applications of sonic energy in industrial processing have increased during the last few years. ${ }^{1}$ The applications of intense sound are based on nonlinear effects produced by finite-amplitude pressure variations. As is wellknown, the propagation of acoustic waves can be described by linear laws only in the case of infinitesimal amplitudes. When the acoustical pressure is of finite amplitude, the equations of motion become nonlinear. This paper deals with the propagation of waves of finite, but moderate, amplitude in fluids.

The propagation of finite-amplitude waves through fluids involves, besides the nonlinear distortion of the waveform, which is the topic of this article, other associated second-order effects such as acoustic radiation pressure, acoustic streaming, and cavitation in liquids. These effects are not treated in this paper.

Classical works can be found in the literature about analytical approximations to describe the nonlinear behavior of progressive waves. ${ }^{2}$ There are several reviews on the development of nonlinear acoustics, ${ }^{3,4}$ and the main contributions in this field were compiled and commented upon by R. T. Beyer in $1984 .^{2}$ A recapitulation on this subject has been recently published by M. F. Hamilton. ${ }^{5}$ Fubini ${ }^{6}$ in 1935 , gave the first explicit solution referred to a plane wave. Analytical models referred to cylindrical and spherical waves can also been found in classical works. ${ }^{7}$ More recently, M. F. Hamilton $^{5}$ and K. E. Froysa ${ }^{8}$ have given analytical solutions for harmonic generation in weakly nonlinear Gaussian beams.

Approximate methods have been used by different authors for solving the nonlinear equations. ${ }^{4}$ The perturbation method, which is used in this paper, is one of the most extended. It has proved to be an efficient tool in the evaluation of nonlinear equations. The description of this method can be widely found in the literature. ${ }^{3-5,9-11}$

The purpose of this work was to develop a numerical method to study the radiated field from high-intensity threedimensional acoustical sources of arbitrary geometry.

Three-dimensional models have been developed referring to linear acoustic fields by using numerical methods. In particular, the finite-element method (FEM) has been widely used to model three-dimensional elastic or piezo-electric radiating structures. ${ }^{12-14}$ Other numerical methods such as the boundary-element method (BEM) ${ }^{15}$ alone or in combination with FEM (BEM-FEM), have also proved to be very powerful for linear radiation modeling. ${ }^{16}$ The interest of extending these tools for realistic three-dimensional modeling of the nonlinear field is evident. This is the goal of the present work.

Nonlinear numerical models of parametric acoustic arrays, as well as piston transducers radiating in water, can be found in the literature ${ }^{17-19}$ In Refs. 17 and 18, the effects of nonlinearity, diffraction, and absorption are all considered of equal order of magnitude. In Ref. 19, a theoretical and experimental study of the near field of an axisymmetric array is presented. Three-dimensional nonlinear propagation models using the angular spectrum method have also been recently published. ${ }^{20}$ In this method, predictions are based upon the measured pressure field at a parallel plane to the source, i.e., modeling needs of some previous experimental knowledge. In a recent review paper written by J. H. Ginsberg and M. F. Hamilton, ${ }^{21}$ about computational methods in nonlinear acoustics, numerical models in the time and frequency domains are presented and discussed. The approximations detailed refer to one-dimensional plane, spherical, and cylindrical waves as well as numerical algorithms for directional three-dimensional waves. These algorithms are based on the 
Khokhlov-Zabolotskaya-Kuznetsov and nonlinear progressive-wave equations, which are only valid for sources in which the characteristic dimension $a$ verifies $k a \gg 1 \quad(k$ is the wave number).

In this article, a finite element three-dimensional model of the nonlinear propagation of acoustic waves is proposed. No restriction on the source geometry and/or vibration form is imposed. The model is implemented in the code ATILA initially developed by Decarpigny et al. ${ }^{12-14}$ for studying linearly elastic and piezo-electric radiating structures. The procedure basically consists of applying a perturbation method to linearize the equations and then using the classical finiteelement method. Second-order approximation is developed; this is the only restriction of the method. The numerical model is validated by comparing with analytical results obtained for several simple specific cases. In addition, experimental validation of the method is carried out by modeling and measuring the high-intensity acoustic field radiated by a stepped-plate transducer.

\section{DEVELOPMENT OF THE NUMERICAL MODEL}

In this section, the numerical method developed to model the three-dimensional propagation of finite, but moderate, amplitude acoustic waves is presented. Only wave propagation in unlimited media is considered. The theoretical basis and its limitations are presented first; the numerical method is detailed afterwards.

\section{A. Theory: The fundamental equations of the problem. A method of successive approximations}

The study considers finite-amplitude progressive, initially harmonic, waves in a homogeneous fluid. Only secondorder terms in the parameter $\left(p_{\mathrm{ac}} / \rho_{0} c_{0}^{2}\right.$ ) (acoustic Mach number) are considered, $p_{\text {ac }}$ being the acoustic pressure, $\rho_{0}$ the density at the instant at which the medium is at rest and undeformed, and $c_{0}$ the small-signal sound speed. Lagrangian coordinates are considered.

In order to obtain a three-dimensional second-order wave equation, the isentropic equation of state and equations expressing the conservation of mass (the continuity equation) and momentum are considered. ${ }^{3,4}$ These equations can be respectively formulated as follows:

$$
\begin{aligned}
& p=P\left(\frac{\rho}{\rho_{0}}\right)^{\gamma}-Q, \\
& \frac{\rho_{0}-\rho}{\rho}=\nabla \cdot \mathbf{u}, \\
& \rho_{0} \frac{\partial^{2} \mathbf{u}}{\partial t^{2}}=-\nabla p,
\end{aligned}
$$

where $p$ is the pressure, $\rho$ is the density of the fluid, $\mathbf{u}$ is the displacement, $\gamma$ is the ratio of specific heats, $P=\left(\rho_{0} c_{0}^{2} / \gamma\right)$ and $Q$ is a constant depending on the considered fluid. Dissipation mechanisms are ignored because only free-field propagation and not very long distances are considered. ${ }^{22}$

Equation (1) can be approximated by expanding it in a Taylor series, retaining terms up to second order

$$
\rho=\rho_{0}+a_{1}\left(p-p_{0}\right)+\frac{a_{2}}{2}\left(p-p_{0}\right)^{2}
$$

where $a_{1}=\left(1 / c_{0}^{2}\right)=\left(\rho_{0} / \gamma P\right), a_{2}=-\left(P \gamma(\gamma-1) / \rho_{0}^{2} c_{0}^{6}\right)$, and $\left(p-p_{0}\right)$ is the acoustic pressure.

From Eqs. (4), (2), and (3), we obtain the second-order wave equation

$$
-\frac{1}{c_{0}^{2}} \frac{\partial^{2} p}{\partial t^{2}}+\nabla^{2} p=-\frac{\beta}{\rho_{0} c_{0}^{4}} \frac{\partial^{2}\left(p-p_{0}\right)^{2}}{\partial t^{2}},
$$

where $\beta=(\gamma+1) / 2$ is the nonlinearity parameter of the fluid which determines the nonlinear characteristic of the propagation medium. For common fluids, the value of this parameter can be found in the literature (see Ref. 3, for example). The parabolic wave Eq. (5) is consistent with the expression proposed by Westervelt ${ }^{23}$ and the one used by Garret et al. ${ }^{19}$

To solve the second-order Eq. (5), the successive approximations method was applied. This method assumes a solution for the acoustic pressure consisting of the addition of two terms in the form

$$
p-p_{0}=p_{1}+p_{2},
$$

where $p_{1}$ represents the first-order approximation and $p_{2}$ the second-order correction, $p_{2}$ being much smaller than $p_{1}\left(p_{2}\right.$ $\left.\ll p_{1}\right)$. Neglecting the terms of third or higher orders, from Eq. (6), it can be written

$$
\left(\frac{p-p_{0}}{\rho_{0} c_{0}^{2}}\right)^{2} \cong\left(\frac{p_{1}}{\rho_{0} c_{0}^{2}}\right)^{2} \text {. }
$$

Substituting Eq. (6) in Eq. (5), and taking into account that the linear solution $p_{1}$ verifies

$$
-\frac{1}{c_{0}^{2}} \frac{\partial^{2} p_{1}}{\partial t^{2}}+\nabla^{2} p_{1}=0
$$

the following equation is obtained for the second-order correction, $p_{2}$ :

$$
-\frac{1}{c_{0}^{2}} \frac{\partial^{2} p_{2}}{\partial t^{2}}+\nabla^{2} p_{2}=-\frac{\beta}{\rho_{0} c_{0}^{4}} \frac{\partial^{2} p_{1}^{2}}{\partial t^{2}} .
$$

Considering the particular case of an initially generated harmonic wave,

$$
p_{1}=\cos (\omega t-\mathbf{k r}) f(\mathbf{r}),
$$

and making use of Eq. (9), Eq. (8) becomes

$$
\frac{4 \omega^{2}}{c_{0}^{2}} P_{2}+\nabla^{2} P_{2}=\frac{2 \omega^{2} \beta}{\rho_{0} c_{0}^{4}} P_{1}^{2},
$$

where $p_{1}=e^{j \omega t} P_{1}$ and $p_{2}=e^{j 2 \omega t} P_{2}$. Equation (10) is a linear nonhomogeneous equation which can be solved by classical methods. For the simplest cases (plane, spherical, or cylindrical waves) it can be solved analytically. However, when acoustical fields of more complex geometry are considered, numerical evaluation becomes necessary. A numerical solution of this equation based on the finite-element method is proposed to be able to solve three-dimensional acoustical problems of arbitrary geometry.

First, the physical limitations of the theory are considered. The first and most important limitation derives from the 
TABLE I. Values of the second- and third-order terms in water for different acoustic pressure levels. $p_{\mathrm{ac}}$ is the pressure level expressed in $\mathrm{dB}$ (Ref. $P_{0}$ $\left.=10^{-6} \mathrm{~Pa}\right), p_{\mathrm{ac}} / \rho_{0} c_{0}^{2}$ is the ratio between the second order and the linear terms, and $\left(p_{\mathrm{ac}} / \rho_{0} c_{0}^{2}\right)^{2}$ is the ratio between the third order and the linear one.

\begin{tabular}{lrrrr}
\hline \hline \multicolumn{5}{c}{$\mathrm{dB}$} \\
\hline$p_{\mathrm{ac}}$ & 180 & 220 & 250 & 300 \\
$p_{\mathrm{ac}} / \rho_{0} c_{0}^{2}$ & -127 & -87 & -56.5 & -7 \\
$\left(p_{\mathrm{ac}} / \rho_{0} c_{0}^{2}\right)^{2}$ & -254 & -174 & -113 & -14 \\
\hline \hline
\end{tabular}

second-order approximation, which prescribes the moderate perturbation condition and neglects all terms of third or higher order. In Table I, the values of the second- and thirdorder terms for different pressure levels in water are compared with the values of the linear term. Thus, for a given acoustical pressure, $p_{\mathrm{ac}}$, the values of the second-order term divided by the linear one, $p_{\mathrm{ac}} / \rho_{0} c_{0}^{2}$, as well as the values of the third-order term divided by the linear one, $\left(p_{\mathrm{ac}} / \rho_{0} c_{0}^{2}\right)^{2}$, are reported. All quantities are expressed in $\mathrm{dB}$ (reference pressure $1 \mu \mathrm{Pa})$.

From this table, the wide application range of the procedure in the case of the water can be noted. Even for very high pressure values $(250 \mathrm{~dB})$, the neglected term remains much smaller than the fundamental (113 dB smaller).

The same calculations carried out for air are displayed in Table II (reference pressure $20 \mu \mathrm{Pa}$ ). For this case, the acoustic Mach number increases faster, so the perturbations are relatively significant even if the absolute values of the pressure are smaller. Thus, the range of application of the proposed method depends on the fluid considered. The value of the nonlinearity parameter, which has not been taken into account in this evaluation, is of the same order of magnitude for both fluids (3.5 for water and 1.3 for air).

A well-known feature of the propagation of a finiteamplitude wave is the increase of the wave distortion with distance. $^{6}$ Even for low-amplitude waves, the high-order components reach a certain intensity if the distance to the source is sufficiently long. This fact gives a spatial limitation for the application of the present approximation. In fact, it is only valid for distances much shorter than the discontinuity distance (distance for the formation of a shock wave). A more precise estimation could be given considering, specifically, the different types of waves. As an example of distances of applicability, the amplitude of the third-order component for a plane wave is calculated and compared with the linear and second-order component generated with a pressure amplitude of $150 \mathrm{~dB}$ (ref. $2 \times 10^{-5} \mathrm{~Pa}$ ) in air at $23600 \mathrm{~Hz}$. Results show that for this case, where the nonlinearities grow

TABLE II. Values of the second- and third-order terms in water for different acoustic pressure levels. $p_{\text {ac }}$ is the pressure level expressed in $\mathrm{dB}$ (Ref. $\left.P_{0}=2 \times 10^{-5} \mathrm{~Pa}\right), p_{\mathrm{ac}} / \rho_{0} c_{0}^{2}$ is the ratio between the second order and the linear terms, and $\left(p_{\mathrm{ac}} / \rho_{0} c_{0}^{2}\right)^{2}$ is the ratio between the third order and the linear one.

\begin{tabular}{lcccc}
\hline \hline \multicolumn{5}{c}{$\mathrm{dB}$} \\
\hline$p_{\mathrm{ac}}$ & 160 & 170 & 180 & 190 \\
$p_{\mathrm{ac}} / \rho_{0} c_{0}^{2}$ & -37.5 & -27 & -17.5 & -7.5 \\
$\left(p_{\mathrm{ac}} / \rho_{0} c_{0}^{2}\right)^{2}$ & -75 & -54 & -35 & -15 \\
\hline \hline
\end{tabular}

very quickly, at $12 \mathrm{~cm}$ from the source we have a third order 38 times smaller than the linear one, while the second-order one is only 6 times smaller.

However, this increase of the distortion is, of course, limited by the dissipation of acoustical energy, which affects the higher harmonics much more. The attenuation effect leads to the saturation of the acoustical waves.

\section{B. Numerical method}

The finite-element method is not detailed here because its general description can be found in many books (see, for example, Ref. 24). The finite-element formulation of the fluid structure problem leads to the following set of equations (see the Appendix):

$$
\left[\begin{array}{cc}
{\left[K_{u u}\right]-\omega^{2}\left[M_{s}\right]} & -[L] \\
\rho^{2} c^{2} \omega^{2}[L]^{T} & {[H]-\omega^{2}[M]}
\end{array}\right]\left[\begin{array}{c}
\widetilde{U} \\
\widetilde{P}
\end{array}\right]=\left[\begin{array}{c}
\widetilde{f} \\
\rho c^{2} \widetilde{\Psi}
\end{array}\right],
$$

where $\left[K_{u u}\right]$ and $\left[M_{s}\right]$ are, respectively, the solid stiffness and consistent mass matrices, $\widetilde{U}$ is the vector of the nodal values of the solid displacement field, $\tilde{f}$ is the vector of the nodal values of the applied forces, $\widetilde{P}$ is the vector of the nodal values of the pressure field, $\widetilde{\Psi}$ is the vector of the nodal values of the pressure-normal derivative on the fluid domain boundary, $[L]$ is the interface connectivity matrix, $[H]$ and $[M]$ are, respectively, the fluid stiffness and consistent mass matrices, and the superscript $T$ means transposed.

The finite-element modeling of a radiating structure is performed by surrounding this structure with a limited fluid domain, upon the external boundary of which a nonreflection condition is prescribed for the acoustic field. This condition is implemented with the help of damping elements which are attached to the external boundary.

Second-order corrections in the radiated acoustic field, according to the theory developed in Sec. I A, imply modifications of the matricial Eq. (11). In Eq. (10), the nonlinear behavior of the fluid is represented by the right-hand side term $\left(2 \omega^{2} \beta / \rho_{0} c_{0}^{4}\right) P_{1}^{2}=f_{B}$, which mathematically is equivalent to a mass source term in the fluid. ${ }^{25}$

The application of the weighted residual method to Eq. (10) leads to the following integral equation: ${ }^{24}$

$$
\begin{gathered}
\iiint_{\Omega_{f}}\left(\frac{\partial \nu}{\partial x_{i}} \frac{\partial p_{2}}{\partial x_{i}}-4 k^{2} \nu p_{2}-\frac{2 \omega^{2} \beta \nu p_{1}^{2}}{\rho_{0} c_{f}^{4}}\right) d \Omega_{f} \\
-\iint_{\Gamma_{p} \cup \Gamma_{\psi} \cup \Gamma_{\infty}} \nu \Psi_{2} d \Gamma=0,
\end{gathered}
$$

where $\nu$ is an arbitrary scalar function. In the following, $\nu$ $=p_{2}$ is chosen.

When discretization is applied, Eq. (12) is written

$$
\sum_{e}\left\{\frac{1}{\rho_{0} c_{f}^{2}} \widetilde{P}_{2}^{e T}\left(\left[H^{e}\right]-\omega^{2}\left[M_{l}^{e}\right]\right) \widetilde{P}_{2}^{e}-\widetilde{P}_{2}^{e T} \widetilde{\Psi}_{2}^{e}-\widetilde{P}_{2}^{e T} \widetilde{R}_{B}^{e}\right\}=0
$$

with

$$
\widetilde{R}_{B}^{e}=-\frac{2 \omega^{2} \beta}{\rho_{0} c_{f}^{4}} \iiint_{\Omega_{f}^{e}}\left[N_{p}^{e}\right]\left[N_{p}^{e}\right] \widetilde{P}_{1}^{e}\left[N_{p}^{e}\right] \widetilde{P}_{1}^{e} d \Omega_{f}^{e} .
$$


After assembling and applying the variational principle, the final expression is obtained

$$
\left\{[H]-4 \omega^{2}\left[M_{l}\right]\right\} \widetilde{P}_{2}=\rho_{0} c_{f}^{2} \widetilde{\Psi}_{2}+\rho_{0} c_{f}^{2} \widetilde{R}_{B} .
$$

In this equation, only the fluid terms of Eq. (11) are included because nonlinearities are considered only in the fluid medium, i.e., linear behavior is assumed for the acoustical source. $\widetilde{P}_{2}$ is a vector that contains the nodal values of the second-order component of the pressure field. The $\widetilde{P}_{1}$ vector is calculated using Eq. (11). After combining Eqs. (11) and (13b), we obtain the loading term $\widetilde{R}_{B}$, which can be introduced into Eq. (14) to calculate $\widetilde{P}_{2}$. The same boundary conditions for the linear modeling are considered, including the nonreflection condition upon the external boundary (see the Appendix).

The FEM solution is implemented in FORTRAN. On an HP workstation (9000/735, RISC $99 \mathrm{MHz})$, the runtimes for an axisymmetrical problem using parabolic elements (15 118 degrees of freedom) are $340 \mathrm{~s}$ for the calculation of the linear solution and $103 \mathrm{~s}$ for the calculation of the second-order correction.

\section{ANALYTICAL VALIDATION OF THE NUMERICAL MODEL}

In this section, an analytical validation of the model for the nonlinear propagation of some well-defined acoustic waves is presented. The results from the analytical study are compared with the numerical results in order to verify the validity of the new method proposed in this paper.

\section{A. Analytical models for plane, spherical, and cylindrical waves}

\section{Plane waves}

The simplest and best-studied case is the plane wave propagation. Various explicit solutions can be found in the literature (for example, an extensive and very complete theoretical treatment of plane progressive sound waves was made by Blackstock ${ }^{26}$ ). The successive approximations method used here can be found in Ref. 6 .

For harmonic excitation of amplitude $P_{0}$, the acoustic pressure is assumed, according to Eq. (6), as an addition of two terms which have the form

$$
p_{1}=P_{0} e^{j(\omega t-k x)}
$$

for the linear approximation, and

$$
p_{2}=-\frac{\beta P_{0}^{2}}{4 \rho_{0} c_{0}^{2}} e^{j 2(\omega t-k x)}(1+j 2 k x)
$$

for the second-order correction.

The amplitude of the second harmonic increases linearly with increasing distance from the source. Consequently, the sinusoidal progressive wave is distorted because of the addition of the increasing second harmonic to the fundamental during propagation.

\section{Spherical waves}

A rigid sphere of radius $r_{0}$ whose surface undergoes constant amplitude harmonic oscillations is considered as a source of waves of pressure amplitude $P_{0}$. The well-known solution of the linear wave equation with these boundary conditions is: ${ }^{22}$

$$
P_{1}=P_{0} r_{0} \frac{e^{-j k\left(r-r_{0}\right)}}{r} .
$$

Writing the wave Eq. (10) in spherical coordinates and inserting (17) in the nonhomogeneous term, the following wave equation for the second-order correction, $P_{2}$, is obtained:

$$
\frac{4 \omega^{2}}{c_{0}^{2}} P_{2}+\nabla^{2} P_{2}=\frac{2 \beta k^{2} P_{0}^{2} r_{0}^{2}}{\rho_{0} c_{0}^{2}} \frac{e^{-j 2 k\left(r-r_{0}\right)}}{r^{2}} .
$$

The following particular solution for Eq. (18) is proposed:

$$
P_{2}=\left(A \frac{\log r / r_{0}}{r}+\sum_{n=2}^{\infty} \frac{C_{n}}{r^{n}}\right) e^{-j 2 k\left(r-r_{0}\right)},
$$

where $A$ and $C_{n}$ are indefinite constants. Substituting (19) into Eq. (18) leads to

$$
A=j \frac{\beta P_{0}^{2} r_{0}^{2} k}{2 \rho_{0} c_{0}^{2}}, \quad C_{n}=\frac{(-1)^{n}(n-2) !}{(j 4 k)^{n-1}} A .
$$

For $r \geqslant 3 \lambda$, all the terms in the series

$$
\sum_{n=2}^{\infty} \frac{C_{n}}{r^{n}}
$$

are much smaller than the principal term

$$
A \frac{\log r / r_{0}}{r}
$$

this series can then be neglected. The condition of rigid sphere

$$
\left(\left[\frac{\partial P_{2}}{\partial r}\right]_{r=r_{0}}=0\right)
$$

implies

$$
P_{2}=A\left(\log \frac{r}{r_{0}}+B\right) \frac{e^{-j 2 k\left(r-r_{0}\right)}}{r},
$$

where $B=1 /\left(1+j 2 k r_{0}\right)$.

According to Eq. (6), the second-order solution for a spherical wave is written as

$$
p=P_{1} e^{j \omega t}+P_{2} e^{j 2 \omega t} .
$$

From Eq. (21), we see that the second harmonic for spherical wave propagation decreases with the distance from the source. However, the ratio between second harmonic and fundamental increases with that distance. 


\section{Cylindrical waves}

A rigid cylinder of infinite length and radius $r_{0}$ acting as a harmonic acoustic source of pressure amplitude $P_{0}$ is considered. The well-known linear solution is ${ }^{22}$

$$
P_{1}=A\left[J_{0}(k r)-j Y_{0}(k r)\right],
$$

where

$$
A=\frac{P_{0}}{J_{0}\left(k r_{0}\right)-j Y_{0}\left(k r_{0}\right)},
$$

$J_{0}$ is the cylindrical Bessel function of the first kind, and $Y_{0}$ is the cylindrical Bessel function of the second kind. At distances from the cylinder larger than a wavelength, the Eq. (23) can be approximated by ${ }^{22}$

$$
P_{1} \cong A \sqrt{\frac{2}{k \pi r}} e^{-j(k r-\pi / 4)} .
$$

The second-order wave equation in cylindrical coordinates is written as

$\frac{4 \omega^{2}}{c_{0}^{2}} P_{2}+\frac{1}{r} \frac{d}{d r} P_{2}+\frac{d^{2}}{d r^{2}} P_{2}=\frac{4 \beta k A^{2}}{\pi \rho_{0} c_{0}^{2}} \frac{e^{-j 2(k r-\pi / 4)}}{r}$. posed:

The following particular solution for Eq. (25) is pro-

$$
P_{2}=f(r) \frac{e^{-j 2 k r}}{\sqrt{r}} .
$$

Substituting Eq. (26) in Eq. (25) and neglecting all the terms of the order $1 /(k r)^{5 / 2}$ leads to

$$
\begin{aligned}
P_{2}= & -C \frac{e^{-j 2 k r}}{j 4 k}\left(2+\frac{1}{j 4 k r}-\frac{1}{2(j 4 k r)^{2}}\right) \\
& -C_{2} \frac{e^{j 2 k r}}{j 4 k \sqrt{r}}+C_{3} \frac{e^{-j 2 k r}}{\sqrt{r}},
\end{aligned}
$$

where

$$
C=\frac{4 \beta k A^{2}}{\pi \rho_{0} c_{0}^{2}} e^{j(\pi / 2)}
$$

and $C_{2}$ and $C_{3}$ are two integration constants to be determined from the boundary conditions. Since only progressive waves are considered here, $C_{2}$ is set to zero. $C_{3}$ is calculated by prescribing boundary conditions (rigid cylinder). The total second-order solution is written as in Eq. (22).

For the cylindrical wave, we observe an intermediate behavior between plane and spherical waves: the second harmonic is almost constant while the fundamental decreases slower than for a spherical wave. The ratio between second harmonic and fundamental amplitude increases with the distance to the source.

\section{B. Numerical-analytical comparison}

By using the previous analytical model, the pressure distribution of the fundamental and second harmonic for plane, spherical, and cylindrical waves in water is calculated. These results are displayed in Fig. 1. As can be easily seen, the proportion of harmonics always increases with the distance

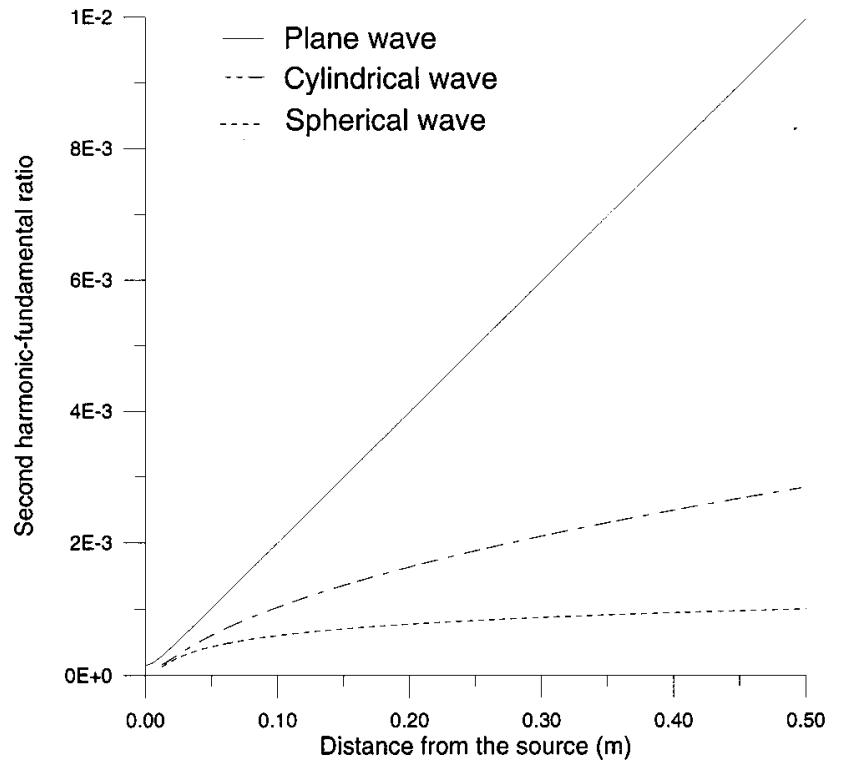

FIG. 1. Second harmonic-fundamental ratio versus distance from the source for progressive plane, spherical, and cylindrical waves in water $(\beta=3.5)$. Initial pressure $P_{0}=4 \times 10^{5} \mathrm{~Pa}$, frequency $f=15000 \mathrm{~Hz}$. (Reference pressure, $10^{-6} \mathrm{~Pa}$.)

to the source, and this increase is less important in the waves in which, for the same vibration amplitude of the source, the acoustic energy density is lower.

The new numerical model is applied for the calculation of the fundamental and second harmonic in plane, spherical, and cylindrical wave propagation. All calculations are made with the assumptions and limitations above mentioned.

Figure 2 shows a comparison between analytical and numerical results for the fundamental and second harmonic pressure amplitudes of a plane wave. It is apparent that excellent agreement is obtained.

The comparison between the analytical and numerical

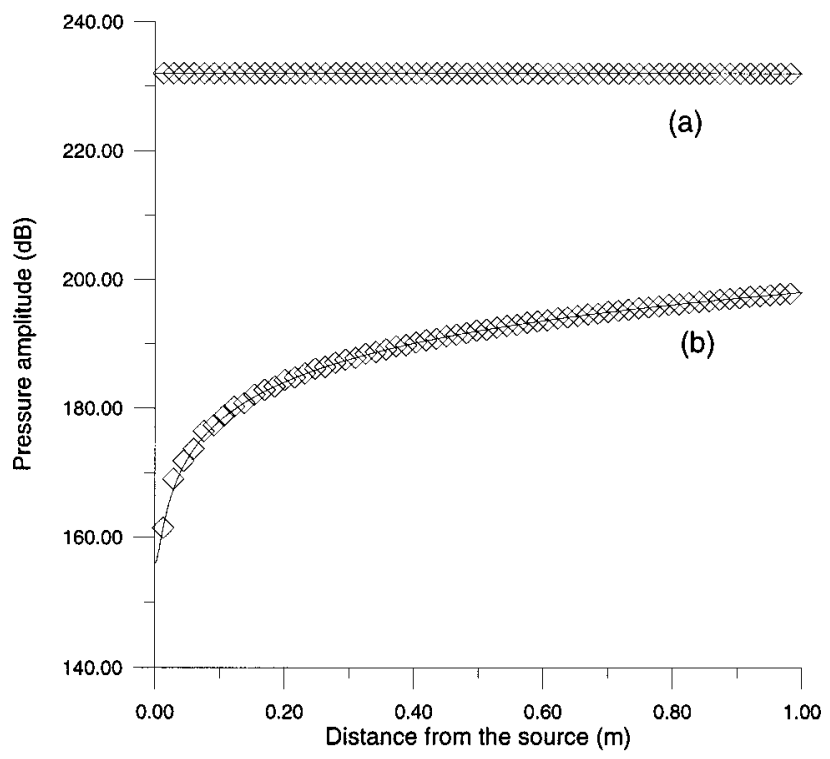

FIG. 2. Comparison between numerical $(\diamond)$ and analytical $(-)$ results for a plane wave in water $(\beta=3.5)$. (a) Fundamental. (b) Second harmonic. Initial pressure $P_{0}=4 \times 10^{5} \mathrm{~Pa}$, frequency $f=15000 \mathrm{~Hz}$. (Reference pressure, $10^{-6} \mathrm{~Pa}$.) 


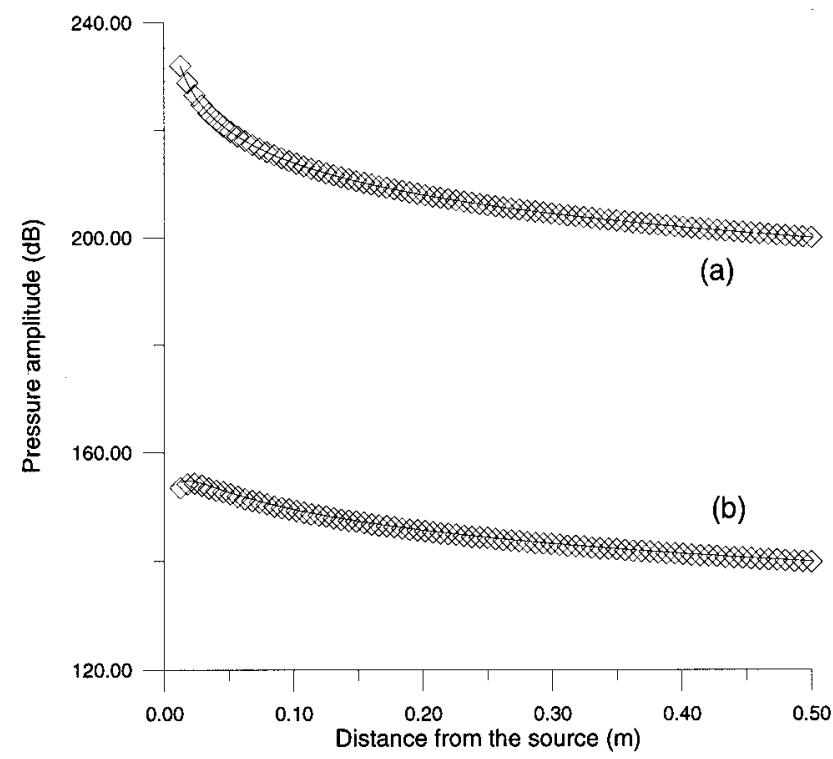

FIG. 3. Comparison between numerical $(\diamond)$ and analytical $(\longrightarrow)$ results for a spherical wave in water $(\beta=3.5)$. (a) Fundamental. (b) Second harmonic. Initial pressure $P_{0}=4 \times 10^{5} \mathrm{~Pa}$, frequency $f=15000 \mathrm{~Hz}$. (Reference pressure, $10^{-6} \mathrm{~Pa}$.)

results for a spherical wave is shown in Fig. 3. Again, it is clear that a very good agreement is obtained.

The analytical-numerical comparison for a cylindrical wave is shown in Fig. 4. Once more, the agreement is very good.

\section{EXPERIMENTAL VALIDATION}

The nonlinear propagation of finite-amplitude waves in air is experimentally studied. A high-directional transducer is designed and constructed in order to have a high concentration of energy and thus, observable nonlinear effects.

\section{A. Experimental study}

The experimental setup for measuring frequency components of the acoustic pressure at different points is shown in Fig. 5. It mainly consists of a power transducer with an elec-

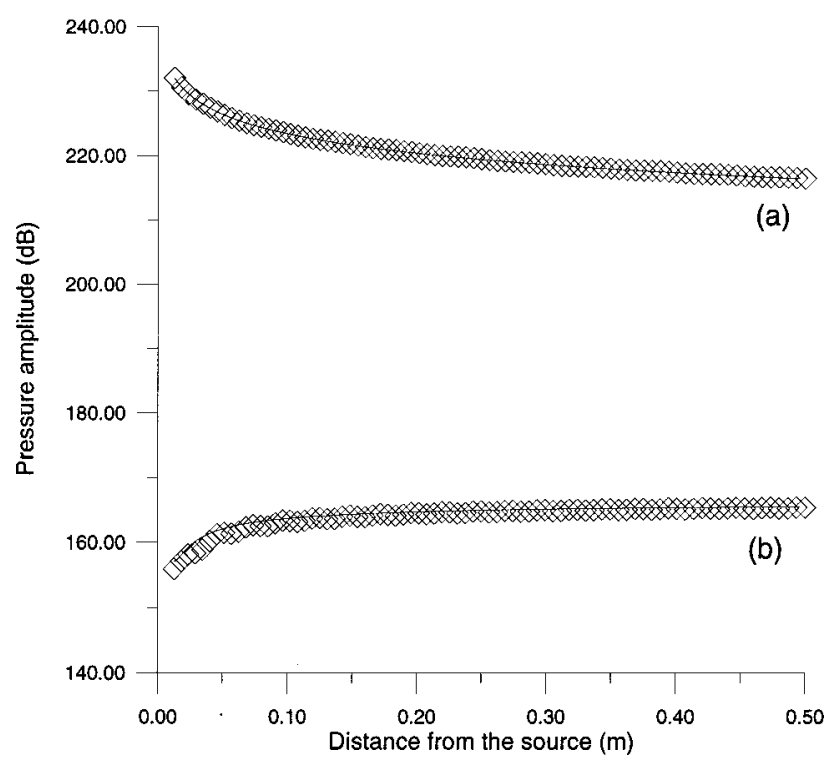

FIG. 4. Comparison between numerical $(\diamond)$ and analytical $(-)$ results for a cylindrical wave in water $(\beta=3.5)$. (a) Fundamental. (b) Second harmonic. Initial pressure $P_{0}=4 \times 10^{5} \mathrm{~Pa}$, frequency $f=15000 \mathrm{~Hz}$. (Reference pressure, $10^{-6} \mathrm{~Pa}$.)

tronic driving system to generate the acoustical field and data acquisition equipment.

The electronic driving system implements a feedback device to automatically adjust the excitation frequency to the transducer resonance frequency. ${ }^{27}$ The basic structure of the transducer is shown in Fig. 5. It consists essentially of a circular plate of stepped shape driven at its center by a piezoelectric vibrator. The vibrator itself consists of a piezoelectric element of transduction in a sandwich arrangement, and a solid horn which acts as a vibration amplifier. The longitudinal vibration, generated by the transducer element and amplified by the mechanical amplifier, drives the radiating plate, which vibrates flexurally in its first axisymmetrical mode. The radiating plate $(6-\mathrm{cm}$ diameter) is made of titanium alloy $(\mathrm{Ti} 6 \mathrm{Al} 4 \mathrm{~V})$ in order to have intense displacements without ultrasonic fatigue problems. ${ }^{28}$ The elements of

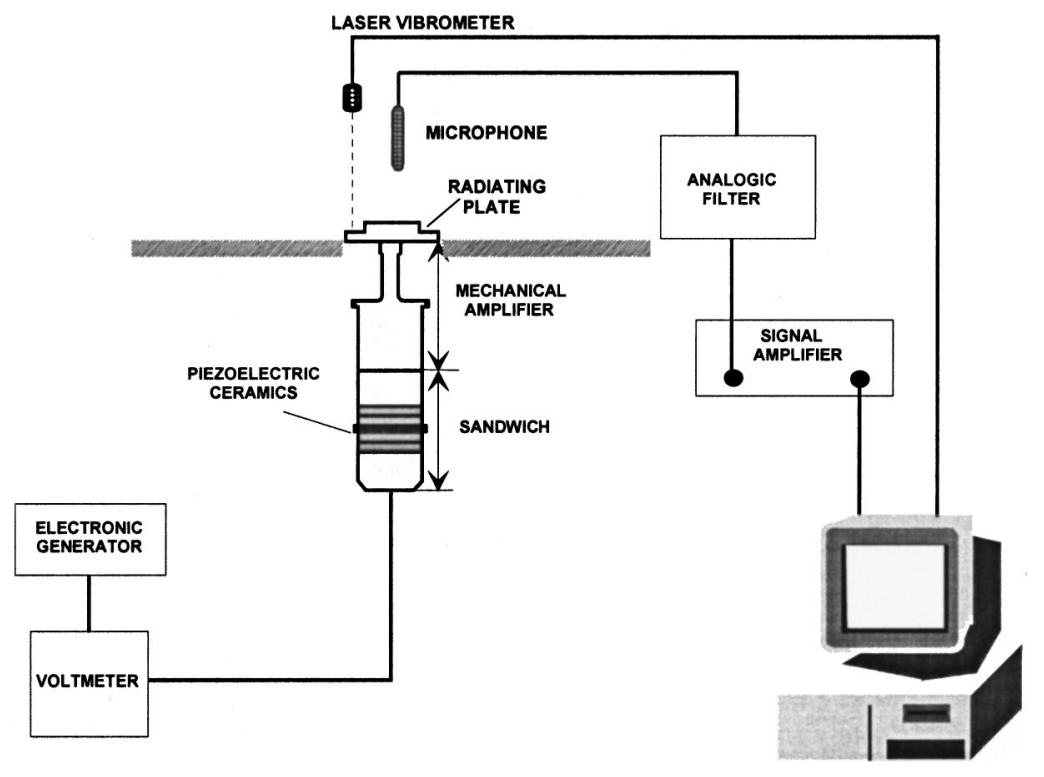

FIG. 5. Experimental setup. 


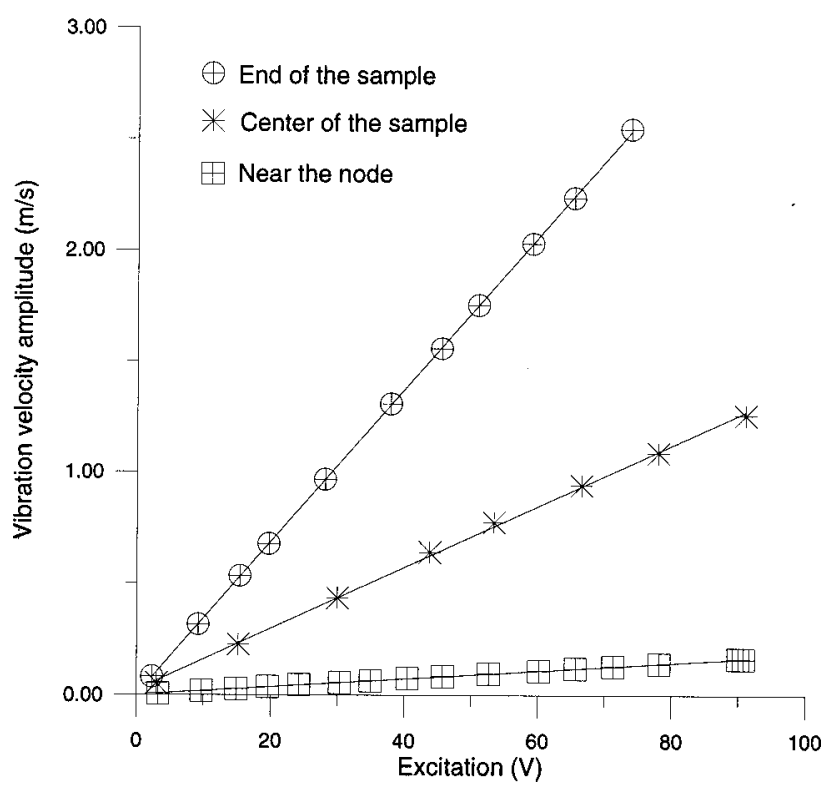

FIG. 6. Vibration velocity versus voltage excitation at different points on the radiator surface. Frequency $f=23600 \mathrm{~Hz}$.

the transducer are calculated to be resonant at the working frequency $(23600 \mathrm{~Hz})$. The specific profile of the plate generates a directional piston-like radiation pattern which allows the production of high-intensity acoustic levels. ${ }^{29}$

The acoustic-pressure distribution and wave shapes are measured by using a Brüel \& Kjaer condenser microphone type 4138 in an anechoic room. An automatic system is used to move the microphone. The signal is stored in a PC and Fourier analysis is performed in order to quantify the amplitudes of the harmonic components.

Measurements of the vibration displacement distribution and wave shape in the radiating plate of the transducer are also carried out by using a laser vibrometer, in order to calibrate it and verify the linearity of its behavior. All measure- ments are carried out in the linear range of the transducer. The obtained results are shown in Fig. 6, which displays the vibration amplitude at different points along a diameter of the plate versus electric excitation. A reasonably linear behavior is observed.

Figure 7 displays the measured acoustic field radiated by the transducer at the fundamental frequency $(23600 \mathrm{~Hz})$. The directional piston-like radiation of the transducer is observed.

Figure 8 shows the measured acoustic-pressure distribution of the second harmonic in the space irradiated by the stepped-plate transducer. The second harmonic radiation pattern is even more directional that the fundamental one. The width of the main lobe is of $12 \mathrm{deg}$ for the fundamental (measured at $-3 \mathrm{~dB}$ from the maximum) and of $7 \mathrm{deg}$ for the second harmonic. It should be noted that this value is not far from the square of the fundamental (7.9 deg) as obtained in Ref. 30.

Because of the piston-like radiation of the transducer, this experimental case is intermediate between plane and spherical waves.

Figures 9 and 10 show the experimental variation of the fundamental and second-harmonic amplitudes as a function of excitation at different points along the acoustic propagation axis (fundamental frequency, $23600 \mathrm{~Hz}$ ). All the measurements are carried out within the near field of the transducer. A linear variation of the fundamental is observed while the second harmonic shows a parabolic increase with excitation. The linear behavior of the fundamental confirms that, in the limits of pressures and distances in which measurements are conducted, the approximation assumed in Eq. (6) is valid. In the experimental working conditions, the discontinuity distance is approximately $50 \mathrm{~cm}$ for plane waves, and on the order of several kilometers for spherical waves (source radius $\left.r_{0}=3 \times 10^{-2}\right) .^{31}$

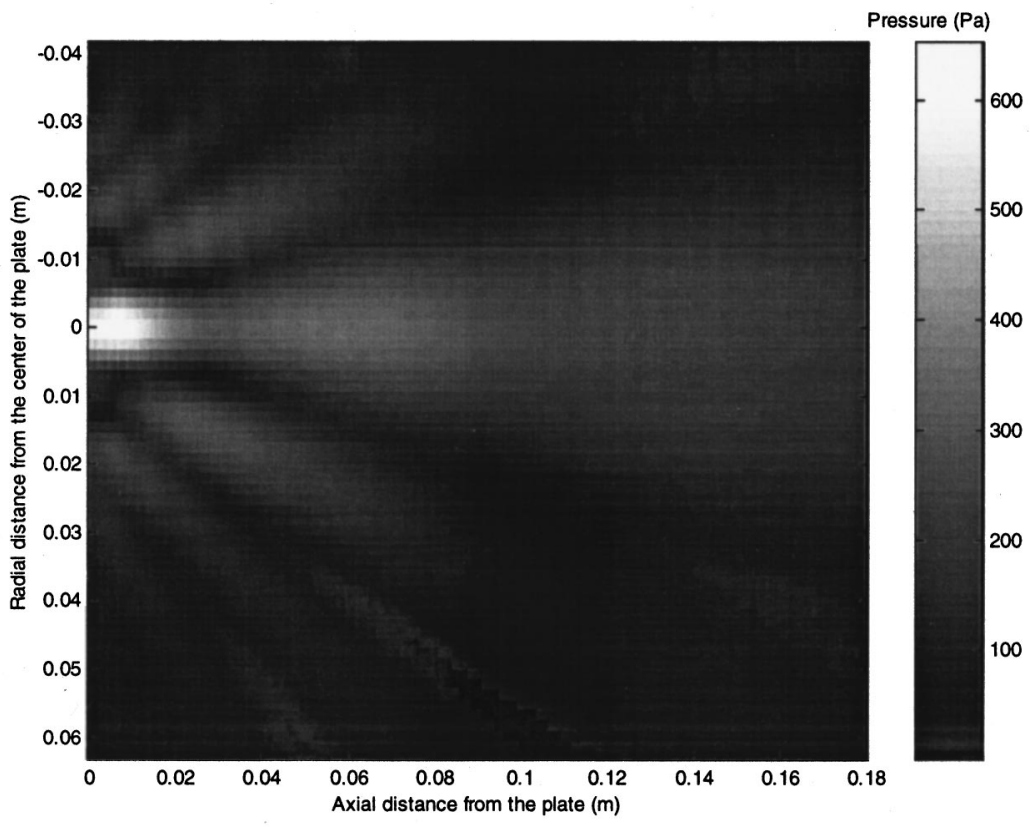

FIG. 7. Measured acoustic field of the transducer at the fundamental frequency $(23600 \mathrm{~Hz})$. Excitation voltage, $50 \mathrm{~V}$. 


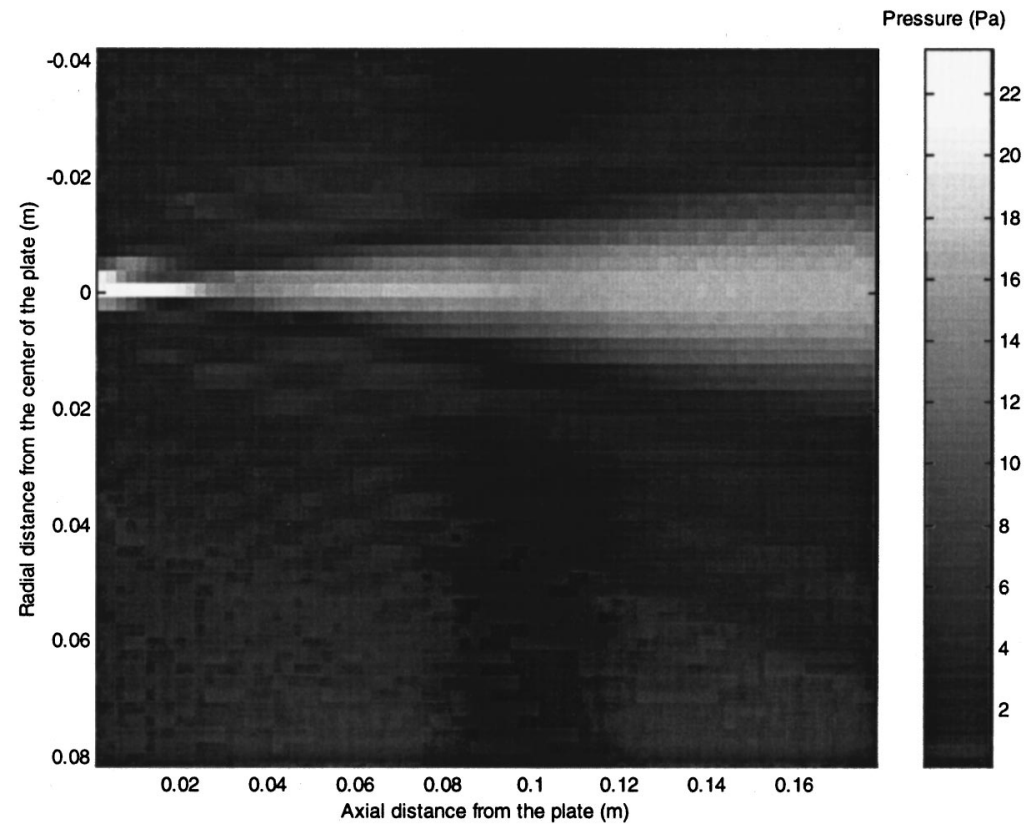

FIG. 8. Measured pressure distribution of the second harmonic $(47200 \mathrm{~Hz})$ in the space irradiated by the transducer. Excitation voltage, $50 \mathrm{~V}$.

\section{B. Numerical-experimental comparison}

To obtain numerical results, the finite-element procedure is applied to the study of the vibrational behavior and the nonlinear radiation of the previously described transducer. The transducer is represented by the radiating plate with a prescribed mechanical excitation at its center. Taking into account the symmetry of the problem, an axisymmetrical analysis is carried out. The finite-element mesh is shown in Fig. 11 where $\Gamma$ represents the external surface. The actual mesh observes the $\lambda / 4$ criterion in air, i.e., it is constituted of parabolic elements smaller than a quarter wavelength of the radiation in the propagation medium. The actual sides of all elements are equal or less than $1.8 \times 10^{-3} \mathrm{~m}$.

Figure 12 shows the comparison of the measured linear

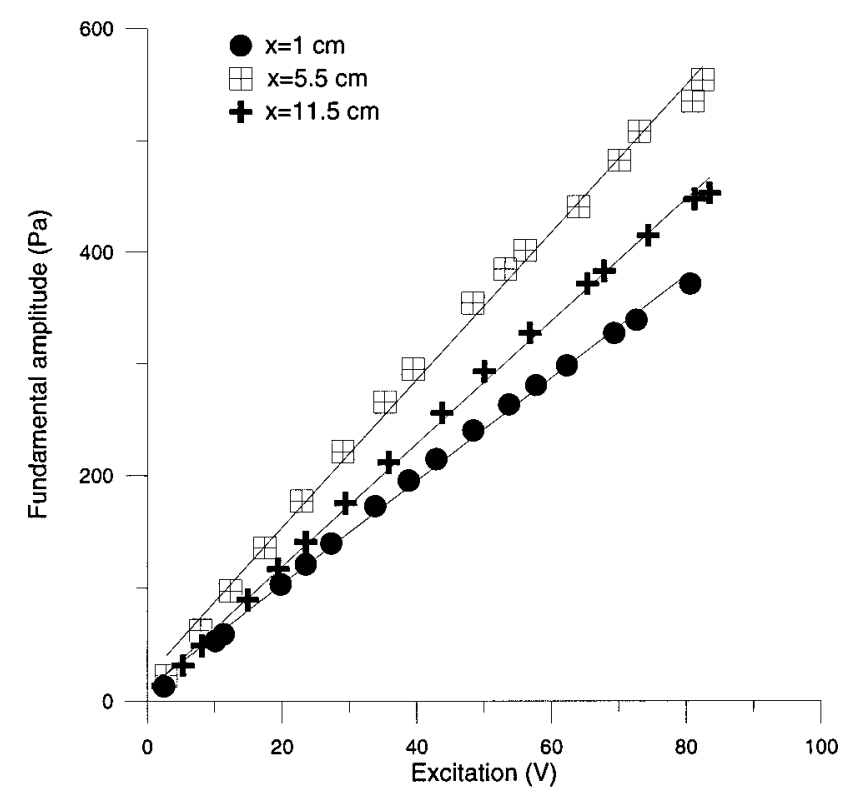

FIG. 9. Measured fundamental pressure amplitude versus excitation at different points along the acoustic axis.

distribution of displacements in the radiating plate of the transducer with the numerical results. The good agreement obtained is a first experimental verification of the validity of the finite-element mesh used. Figure 13 shows the comparison of the numerical and experimental data for the soundpressure distribution of the fundamental and second harmonic along the acoustic axis. The error bars indicate dispersion in the experimental data. The good agreement again obtained represents an experimental confirmation of the validity of the numerical nonlinear modeling method presented here.

\section{CONCLUSIONS}

A numerical method for studying the nonlinear threedimensional propagation of high-intensity acoustic waves

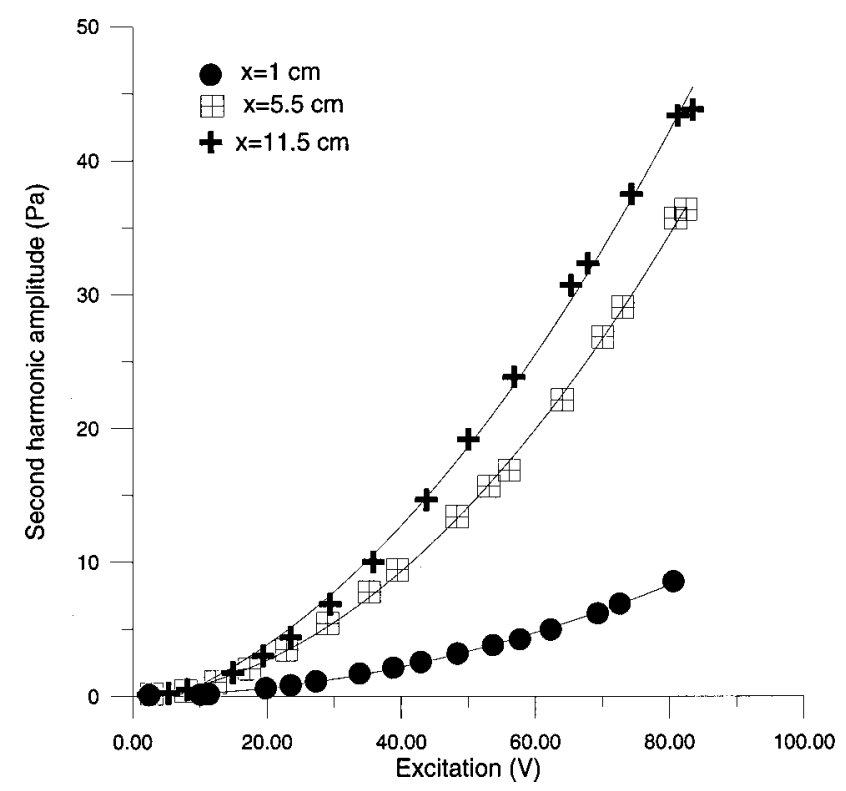

FIG. 10. Measured second-harmonic pressure amplitude versus excitation at different points along the acoustic axis. 


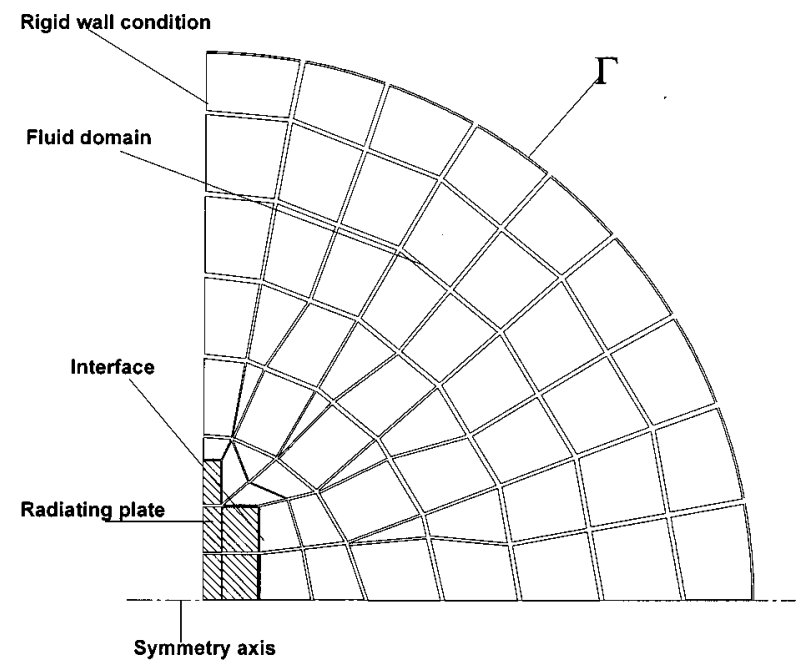

FIG. 11. Scheme of the finite-element mesh.

has been presented. It is based upon a combination of a second-order perturbation theory and the finite-element method. The new method has been applied to different cases, and the obtained results have been compared to analytical as well as experimental results. The good agreement obtained in all cases shows the validity of the numerical method, which allows one to model the nonlinear acoustic field radiated from any real source. The only restrictions are derived from the second-order approximation and computer limitations.

Modeling the nonlinear field of actual transducers opens up new possibilities in transducer design for industrial processing, where high-intensity effects are important and nonlinear behavior cannot be neglected. Thus, the method presented here can be an excellent tool to improve the design of high-power transducers and to analyze the influence of their geometry and elastic features on the nonlinear radiated field. Other applications such as modeling parametric arrays are also potentially interesting.

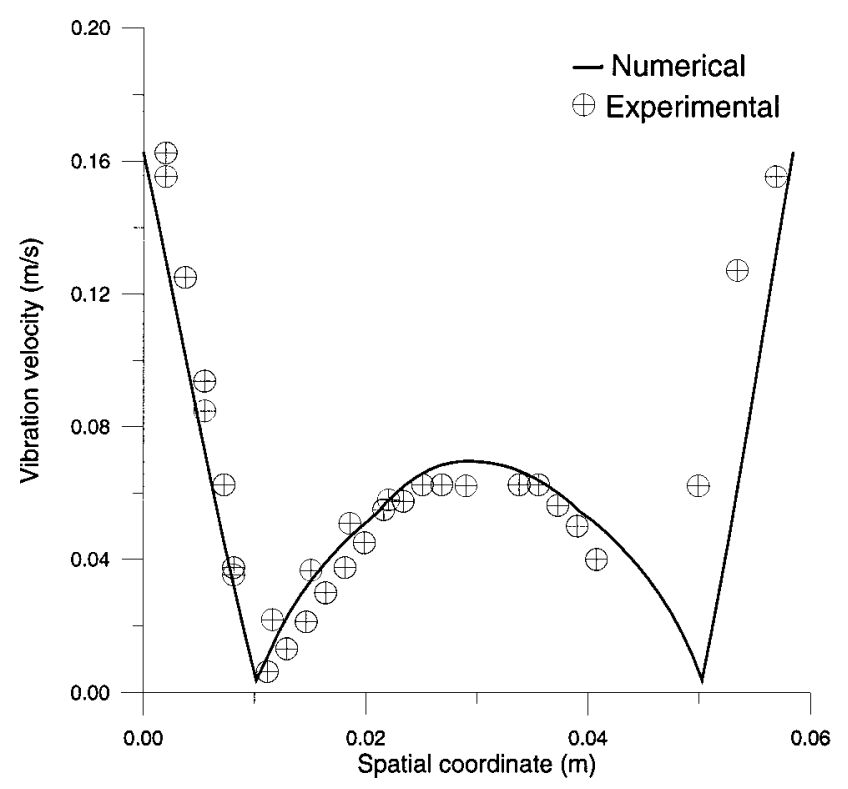

FIG. 12. Experimental-numerical comparison of the vibration velocity distribution along a diameter of the radiating plate.

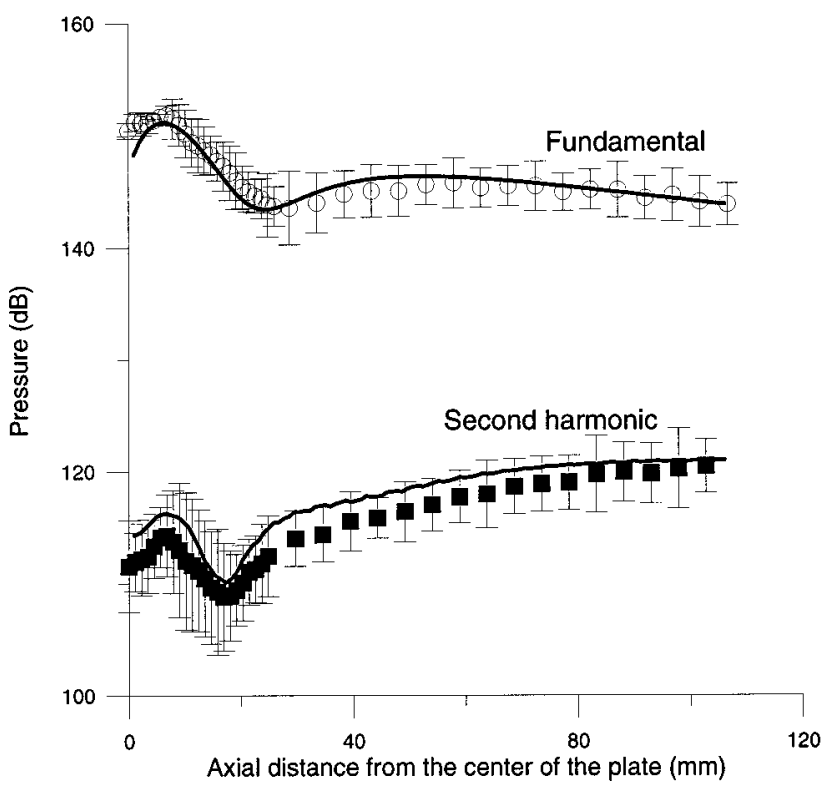

FIG. 13. Experimental numerical comparison of the fundamental and second-harmonic pressure amplitudes along the acoustic axis, expressed in dB (Reference pressure, $2 \times 10^{-5} \mathrm{~Pa}$ ). Fundamental frequency, $23600 \mathrm{~Hz}$.

\section{ACKNOWLEDGMENTS}

This research was supported by a postdoctoral fellowship of the DGICT (State Secretary of Universities and Research) Ref No.: EX94 70735053 and a post-doctoral contract of the CSIC (Higher Council for Scientific Research). The work was developed within the frame of the Projects CICYT-AMB96-A211-CO2 and BRITE/EURAM No. BRPR-CT96-0157. The authors would like to thank Dr. L. Elvira-Segura for his very valuable help in doing experiments.

\section{APPENDIX: FINITE-ELEMENTS FORMULATION OF LINEAR ACOUSTIC WAVE PROPAGATION FOR AN OPEN FLUID}

For a homogeneous, compressible, and infinite fluid $\Omega_{f}$, (sound velocity $c_{f}$ and density $\rho_{f}$ ), described in the frame of linear acoustics, the acoustic pressure $p$ verifies in the frequency domain

$$
\nabla^{2} p+k^{2} p=0,
$$

where $k$ is the wave number. Boundary conditions of Dirichlet type (prescribed pressure on the boundary domain $\Gamma_{p}$ )

$$
p=p^{0},
$$

or of Neumann type (prescribed normal derivative of pressure on the boundary domain $\Gamma_{\Psi}$ )

$$
n_{i} \frac{\partial p}{\partial x_{i}}=\psi^{0},
$$

are considered, $\vec{n}$ being the normal vector to $\Gamma_{\Psi} . \Gamma_{p}$ and $\Gamma_{\Psi}$ are oriented toward the fluid.

In the far field, Sommerfeld's radiation conditions prescribe that $\lim _{r \rightarrow \infty}(r p)$ is finite and 


$$
\lim _{r \rightarrow \infty}\left[r\left(j k p+\frac{\partial p}{\partial r}\right)\right]=0
$$

$r$ being the distance to the acoustical center.

\section{A. Variational formulation and discretization}

When building a finite-element representation of the problem considered, the infinite extension of $\Omega_{f}$ is the main difficulty. The method consists of limiting $\Omega_{f}$ by a surface $\Gamma$ pointing outwards, on which a reasonable boundary condition is prescribed. We consider a spherical surface $\Gamma$ whose radius is large enough to verify Sommerfeld's conditions. Equations (A1), (A2), and (A3) are the Euler equation and the boundary conditions associated to the functional $L_{f}$, respectively,

$$
\begin{aligned}
L_{f}= & \frac{1}{\rho_{0} \omega^{2}} \iiint_{\Omega_{f}} \frac{1}{2}\left[\left(\frac{\partial p}{\partial x_{i}}\right)^{2}-k^{2} p^{2}\right] d \Omega_{f} \\
& -\frac{1}{\rho_{0} \omega^{2}} \iint_{\Gamma_{\rho} \cup \Gamma_{\Psi} \cup \Gamma_{\infty}} p \psi d \Gamma .
\end{aligned}
$$

The solution of the problem is the minimum of $L_{f}$. The spatial discretization is performed by meshing the fluid domain in elements connected by nodes. The pressure $p$ at a point in the element is related to the nodal values of the pressure $\widetilde{P}^{e}$ by the interpolation functions

$$
p=\left[N_{p}^{e}\right] \widetilde{P}^{e},
$$

where $\left[N_{p}^{e}\right]$ is a matrix constructed from the interpolation functions. From (A4) and (A5), $L_{f}$ is written

$$
\begin{aligned}
L_{f}= & \sum_{e}\left\{\frac{1}{2 \rho_{0}^{2} c_{f}^{2} \omega^{2}} \widetilde{P}^{e T}\left(\left[H^{e}\right]-\omega^{2}\left[M^{e}\right]\right) \widetilde{P}^{e}\right. \\
& \left.-\frac{1}{\rho_{0} \omega^{2}} \widetilde{P}^{e T} \widetilde{\Psi}^{e}\right\},
\end{aligned}
$$

where

$$
\begin{aligned}
& {\left[H^{e}\right]=\iiint_{\Omega_{f}^{e}} \rho_{0} c_{f}^{2}\left[B_{p}^{e}\right]^{T}\left[B_{p}^{e}\right] d \Omega_{f}^{e},} \\
& {\left[M^{e}\right]=\iiint_{\Omega_{f}^{e}} \rho_{0}\left[N_{p}^{e}\right]^{T}\left[N_{p}^{e}\right] d \Omega_{f}^{e},} \\
& \tilde{\Psi}^{e}=\iint_{\Gamma^{e}}\left[N_{p}^{e}\right]^{T} \psi
\end{aligned}
$$

and $\left[B_{p}^{e}\right]$ is a matrix obtained from the derivatives of interpolation functions. After minimizing with respect to each nodal value and assembling, the linear system of equations is obtained,

$$
\left\{[H]-\omega^{2}[M]\right\} \widetilde{P}=\rho_{0} c_{f}^{2} \widetilde{\Psi} .
$$

\section{B. Damping elements}

As $\Gamma$ is located in the far field, the pressure and its normal derivative are related by the spherical wave relation

$$
\frac{\partial p}{\partial r}=-\left(\frac{1}{r}+j k\right) p
$$

This condition corresponds to the damping of the monopolar contribution of the outgoing wave. More elaborate conditions absorbing successive components of the pressurefield multipolar expansion are possible. ${ }^{12}$

After spatial discretization, Eq. (A8) is written on $\Gamma$

$$
\widetilde{\Psi}=\frac{1}{\rho_{0} c_{f}^{2}}[G] \widetilde{P} .
$$
tions

Combining (A7) and (A9) leads to the final set of equa-

$$
\left\{[H]-\omega^{2}[M]-[G]\right\} \widetilde{P}=\rho_{0} c_{f}^{2} \widetilde{\Psi}_{\mathrm{int}},
$$

where $\widetilde{\Psi}_{\text {int }}$ denotes nodal values of $\widetilde{\Psi}$ excluding the nodes located on $\Gamma$.

1 J. A. Gallego-Juárez, "Transducer needs for macrosonics," in Power Transducers for Sonics and Ultrasonics, edited by B. Hamonic and J. N. Decarpigny (Springer, Berlin), pp. 35-47 (1990).

${ }^{2}$ R. T. Beyer, "Nonlinear acoustics in fluids," Benchmark Papers in Acoustics Series (Van Nostrand Reinhold, New York, 1984).

${ }^{3}$ L. Bjorno, "Nonlinear acoustics," in Acoustics and Vibration Progress, Vol. 2, edited by R. W. B. Stephens and H. G. Leventhall (Chapman and Hall, London, 1976), pp. 101-198.

${ }^{4}$ R. T. Beyer, "Nonlinear acoustics," in Physical Acoustics, edited by W. P. Mason (Academic, New York, 1965), Vol. II, Part B, pp. 231-332.

${ }^{5}$ M. F. Hamilton, "Nonlinear effects in sound beams," in Encyclopedia of Acoustics, edited by Malcolm J. Crocker (Wiley, New York, 1997), Vol. I, pp. 249-256.

${ }^{6}$ E. Fubini-Ghiron, "Anomalies in the propagation of an acoustic wave of larger amplitude," Alta Freq. 4, 532-581 (1935). English version by R. T. Beyer in Ref. 2.

${ }^{7}$ D. T. Blackstock, "On plane, spherical, and cylindrical sound waves of finite amplitude in lossless fluids," J. Acoust. Soc. Am. 36, 217-219 (1964).

${ }^{8}$ K. E. Froysa, “'Weakly nonlinear propagation of a pulsed sound beam," J. Acoust. Soc. Am. 95, 123-130 (1994)

${ }^{9}$ M. A. Breazeale and J. Philip, in Physical Acoustics, edited by W. P. Mason and R. N. Thurston (Academic, Orlando, 1984), Vol. XVII, pp. $1-60$.

${ }^{10} \mathrm{C}$. Campos-Pozuelo and J. A. Gallego-Juárez, "Finite amplitude standing waves in metallic rods," J. Acoust. Soc. Am. 97, 875-881 (1995).

${ }^{11}$ C. Campos-Pozuelo and J. A. Gallego-Juárez, "Finite amplitude flexural vibrations at ultrasonic frequencies in metallic bars," J. Acoust. Soc. Am. 98, 1742-1750 (1995).

${ }^{12} \mathrm{R}$. Bossut and J-N. Decarpigny, "Finite element modelling of radiating structures using dipolar damping elements,' J. Acoust. Soc. Am. 86, 1234-1244 (1989).

${ }^{13}$ B. Hamonic, "Application of the finite element method to the design of power piezoelectric sonar transducers," in Power Sonic and Ultrasonic Transducers Design, edited by B. Hamonic and J. N. Decarpigny (Springer, Berlin, 1988), pp. 143-159.

${ }^{14}$ B. Hamonic, J. C. Debus, J. N. Decarpigny, D. Boucher, and B. Tocquet, "Analysis of a radiating thin-shell sonar transducer using the finiteelement method," J. Acoust. Soc. Am. 86, 1245-1253 (1989).

${ }^{15}$ A. Lavie, "Modelisation du rayonnement ou de la diffraction acoustique par une méthode mixte équation intégrales-champ nul," Ph.D. thesis, Lille University (1989)

${ }^{16}$ C. Campos-Pozuelo, A. Lavie, B. Dubus, G. Rodríguez-Corral, and J. A. Gallego-Juárez, "Numerical and experimental study of radiated field of high-power ultrasonic transducers for industrial application in gases," Acoustical Society of America/Japan. Third Joint Meeting. J. Acoust. Soc. Am. 100, 2774 (1996).

${ }^{17} \mathrm{~J}$. N. Tjotta and S. Tjotta, "Nonlinear equations of acoustics, with application to parametric acoustic arrays," J. Acoust. Soc. Am. 69, 1742-1750 (1981).

${ }^{18}$ S. N. Nachef, D. Cathignol, J. N. Tjotta, A. M. Berg, and S. Tjotta, 
"Investigation of a high intensity sound beam from a plane transducer. Experimental and theoretical results,' J. Acoust. Soc. Am. 98, 2303-2323 (1995).

${ }^{19}$ G. S. Garret, J. N. Tjotta, and S. Tjotta, "Nearfield of a large acoustic transducer, part II: Parametric radiation,'” J. Acoust. Soc. Am. 74, 10131020 (1983).

${ }^{20}$ C. J. Vecchio and P. A. Lewin, "Finite amplitude acoustic propagation modeling using the extended angular spectrum method,' J. Acoust. Soc. Am. 95, 2399-2408 (1994).

${ }^{21}$ J. H. Ginsberg and M. F. Hamilton, "Computational methods,' in Nonlinear Acoustics, edited by M. F. Hamilton and D. T. Blackstock (Academic, New York, 1998).

${ }^{22}$ P. M. Morse, Vibration and Sound (Acoustical Society of America, New York, 1981).

${ }^{23}$ P. J. Westervelt, "Parametric acoustic array,' J. Acoust. Soc. Am. 35, 535-537 (1963).

${ }^{24}$ O. C. Zienkiewicz, The Finite Element Method, 3rd ed. (McGraw-Hill, London, 1977).
${ }^{25}$ A. D. Pierce, Acoustics (Acoustical Society of America, New York, 1989).

${ }^{26}$ D. T. Blackstock, "Propagation of plane sound waves of finite amplitude in nondissipative fluids,' J. Acoust. Soc. Am. 34, 9-30 (1962).

${ }^{27}$ J. A. Gallego-Juárez, G. Rodríguez-Corral, J. L. San Emeterio, and F. Montoya-Vitini, European Patent EP 450,030 (1991). US Patent 5,299,175 (1994).

${ }^{28}$ C. Campos-Pozuelo and J. A. Gallego-Juárez, "Limiting strain of metals subjected to high-intensity ultrasound,' Acust. Acta Acust. 82, 823-828 (1996).

${ }^{29}$ J. A. Gallego-Juárez, "High power ultrasonic transducers for use in gases and interphases,' in Power Sonic and Ultrasonic Transducers Design, edited by B. Hamonic and J. N. Decarpigny (Springer, Berlin, 1988), pp. 175-184.

${ }^{30}$ J. C. Lockwood, T. G. Muir, and D. T. Blackstock, "Directive harmonic generation in the radiation field of a circular piston,' J. Acoust. Soc. Am. 53, 1148-1153 (1973).

${ }^{31}$ J. A. Shooter, T. G. Muir, and D. T. Blackstock, "AAcoustic saturation of spherical waves in water,' J. Acoust. Soc. Am. 55, 54-62 (1974). 